\title{
The development of the Canberra symptom scorecard: a tool to monitor the physical symptoms of patients with advanced tumours Margherita J Barresi ${ }^{\dagger 1,2,3}$, Bruce Shadbolt ${ }^{\dagger 3}$, Don Byrne ${ }^{\dagger 2}$ and Robin Stuart- Harris* +1
}

Address: ${ }^{1}$ Medical Oncology Unit, The Canberra Hospital, Woden, Canberra, ACT 2606, Australia, ${ }^{2}$ School of Psychology, The Australian National University, Canberra, ACT 0200, Australia and ${ }^{3}$ Centre for Advances in Epidemiology and Information Technology, The Canberra Hospital, Woden, Canberra, ACT 2606, Australia

Email: Margherita J Barresi - margherita.barresi@defence.gov.au; Bruce Shadbolt - bruce.shadbolt@act.gov.au; Don Byrne - don.byrne@anu.edu.au; Robin Stuart-Harris* - robin.stuart-harris@act.gov.au

* Corresponding author †Equal contributors

Published: 17 December 2003

BMC Cancer 2003, 3:32
Received: 27 August 2003

Accepted: 17 December 2003

This article is available from: http://www.biomedcentral.com/I47I-2407/3/32

(C) 2003 Barresi et al; licensee BioMed Central Ltd. This is an Open Access article: verbatim copying and redistribution of this article are permitted in all media for any purpose, provided this notice is preserved along with the article's original URL.

\begin{abstract}
Background: Patients with advanced (incurable) tumours usually experience a diverse burden of symptoms. Although many symptom assessment instruments are available, we examined whether these addressed tumour-related symptoms.

Methods: We reviewed existing symptom assessment instruments and found a number of deficiencies such as instruments being too long or burdensome, too short, or measuring quality of life rather than tumour-related symptoms. Others focused on emotional, rather than physical symptoms. Therefore, we decided to devise a new symptom instrument. A list of 20 symptoms common in patients with advanced tumours generated from the literature and existing instruments, was ranked according to prevalence by 202 Australian clinicians. Following clinicians' responses, the list was revised and two severity assessment scales (functional severity and distress severity) added. The resultant 18-item list was assessed in 44 outpatients with advanced tumours.

Results: Patient responses indicated that a shorter questionnaire of II items, reflecting three main symptom clusters, provided a good representation of physical symptoms. An additional symptom that is an important predictor of survival was added, making a 12-item questionnaire, which was entitled "The Canberra Symptom Scorecard" (CSS). For symptom severity, the distress severity scale was more appropriate than the functional severity scale.

Conclusion: The CSS focuses on tumour-related physical symptoms. It is about to be assessed in patients with advanced tumours receiving palliative treatments, when it will also be validated against existing instruments.
\end{abstract}

\section{Background}

Few metastatic tumours are curable and the principal aim of treatment is to improve patients' tumour-related symptoms to maximise quality of life (QoL). A number of QoL and symptom assessment instruments [1-15] has been developed for use in patients with advanced tumours (Table 1 - see additional file 1 ). These are usually completed by the patient, and predominantly are used as outcome measures in clinical trials [15-22]. In clinical practice, clinicians usually assess symptoms in a less 
formal fashion, although there are instruments, such as the Edmonton symptom assessment system (ESAS) [21] that are used on a regular basis, in some practices.

Most appraisals of existing symptom assessment instruments have concluded that they are relevant and acceptable for QoL assessment in patients with advanced tumours [1,9-13,15,16,18,20-25]. On the other hand, a number of limitations has been identified in existing instruments: many of the instruments are too lengthy to be administered regularly (e.g. the adapted symptom distress scale (ASDS), the functional assessment of cancer therapy scale-general (FACT-G), the McGill quality of life questionnaire (MQOL), the Memorial symptom assessment scale (MSAS), and the Rotterdam symptom checklist (RSCL)) $[6,7,11,12,14]$ or they provide only limited benefit in the assessment of symptoms in the more seriously ill patients (e.g. ESAS, RSCL, the patient evaluated problem scores (PEPS)) $[20,21,24]$. Further, the instrument may fail to measure the patient's experience (e.g. the support team assessment schedule (STAS)) $[9,10]$, or the reference period is either absent or too short (MQOL) $[22,25]$. Those that measure QoL rather than tumourrelated symptoms (e.g. FACT-G, the EORTC quality of life questionnaire (QLQ-C30), MQOL) tend to be too broad and less relevant for measuring symptoms $[7,11]$. In contrast, other instruments are too short to be comprehensive (e.g. PEPS) [24], or there is doubt that the coverage of symptoms is appropriate (e.g. the M.D. Anderson symptom inventory (MDASI)) [13]. There is also inconsistency between the severity assessment scales used by different instruments, typically either indicating the impairment of functioning (e.g. FACT-G) [7], or the distress caused by a symptom or symptoms (e.g. ESAS, the symptom distress scale (SDS), modified SDS, ASDS, MQOL) $[1,3-6,11]$, or both (e.g. MDASI, RSCL) $[13,14]$.

Because of these limitations, we decided to investigate the optimal coverage of symptoms necessary and the most appropriate severity assessment scale in an effort to identify an optimal symptom assessment instrument for patients with advanced tumours.

In this study, we explored patient and clinician prevalence rankings of tumour-related symptoms in outpatients with advanced tumours. Based on these relationships, we identified the shortest list of symptoms that covered the major clusters of tumour-related symptoms best for this population. We also examined two different severity assessment scales for each symptom to assess variation between the scales, and whether more than one type of severity assessment scale was needed.

\section{Methods}

\section{The common symptoms in advanced tumours}

A list of 33 of the most frequent symptoms experienced by patients with advanced tumours was compiled from a review of the literature [26,27] and existing symptom assessment instruments. After interviews on symptom prevalence with 14 patients with advanced tumours and with three clinicians involved in the management of patients with advanced tumours from The Canberra Hospital, the list was reduced to 20 symptoms.

\section{Assessing the initial 20-item symptom list}

The 20-item symptom list was circulated to Medical Oncologists, Radiation Oncologists and Palliative Care Physicians around Australia who were invited to comment on the 20 symptoms included in the list and to rank the symptoms according to prevalence in patients with advanced tumours. Clinicians were identified by their membership of the special society for their subspecialty. Two versions of the symptom list were generated using different ordering and one of the versions was randomly attached to each invitation. Space was also made available for clinicians to add up to three additional symptoms that they believed should also be included. Of the 522 clinicians contacted, 202 (38.7\%) responded, with 184 clinicians ranking all 20 symptoms. These responses were analysed and the number of symptoms included in the list was reduced further.

\section{Assessing the revised symptom list in patients with advanced tumours}

Between April and June 2001, the revised symptom list was assessed in 44 outpatients with advanced tumours attending the Medical Oncology Unit or the Department of Radiation Oncology, at the Canberra Hospital. The oncologists were given details of the study and enrolled eligible patients in a sequential fashion. Eligibility criteria required that patients were aged 18 years or older, had an advanced (incurable) tumour, and were receiving palliative treatment(s). In addition, patients were required to have an ECOG performance status of $0-3$, adequate English language skills to complete the questionnaire, no serious concomitant medical or psychiatric condition, and to give written informed consent to participate. The majority of patients completed the questionnaire on their own, but assistance was available from the research staff, if required.

Details of the 44 outpatients who participated are shown in Table 1 . The majority was male, the median age was 60 years (range 39-81 years) and the patients had a variety of incurable solid tumours or lymphoma. Of the 44 patients, eight remain alive, but the rest have died. The median survival for the group was 39 weeks (range 3 - 128+ weeks). Enrolment was restricted to 44 patients as post hoc power 
Table 2: Demographic and Clinical Characteristics of Patients in the Study.

\begin{tabular}{lc}
\hline Characteristic & Outpatients, $\mathbf{n}=\mathbf{4 4}(\%)$ \\
\hline Median age (range) & $60(39-81)$ years \\
Male & $26(59)$ \\
English as first language & $38(86)$ \\
ECOG performance status 0-I & $30(68)$ \\
Current Treatment(s) & \\
Chemotherapy & $29(66)$ \\
Radiation therapy & $10(22)$ \\
Symptomatic therapy & $15(35)$ \\
Herbal medicine & $3(7)$ \\
Hormone therapy & $2(5)$ \\
Primary Tumour Site & \\
Colorectal cancer & $14(32)$ \\
Non small cell lung cancer & $5(12)$ \\
Breast cancer & $5(12)$ \\
Small cell lung cancer & $2(5)$ \\
Non Hodgkin's lymphoma & $2(5)$ \\
CNS (glioblastoma multiforme) & $2(5)$ \\
Prostate cancer & $2(5)$ \\
Other & $12(24)$
\end{tabular}

calculations indicated that the sample of 44 patients yielded a power of over $90 \%$ to detect moderate to strong symptom relationships at a significance level of 5\%. Generally, both staff and patients found the questionnaire easy to complete, with questionnaire completion taking approximately seven minutes, on average.

Patients' assessments were used to provide a comparison with the clinicians' prevalence ranking of symptoms, and to identify the preferred scale for assessing symptom severity (Table 3 - see additional file 2).

\section{Statistical analysis}

SPSS v10.0 was used to analysis the data. In addition to descriptive statistics, the analysis contained an examination of the prevalence of symptoms among the patients with advanced tumours, and a series of multivariate analyses to obtain a final set of symptoms for inclusion in the CSS. Multi-dimensional scaling was used to examine the relative (Euclidean) distances between symptoms, based on clinicians' prevalence rankings. Spearman's rho correlations were used to determine the relationship between the patients' and clinicians' symptom prevalence rankings.

Linear regression (ordinary least squares) analyses were used to determine the most appropriate scale for assessment of symptom severity (distress versus functional impairment). Composite measures of the two scales were constructed for all the symptoms to assess the explanatory power of each scale, using adjusted variance $\left(\mathrm{R}^{2}\right)$ for the sample size. Data reduction analysis (principal component) was employed using the preferred scale to identify symptom clusters. Components required eigenvalues over 1 and were varimax rotated, to produce the best cluster fit. Stepwise ordinary least squares regression analyses were used to identify symptoms that were redundant within a cluster. Composite measures of the symptoms were constructed for the clusters to assess the explanatory power of each symptom, using adjusted $\mathrm{R}^{2} \mathrm{~s}$. Entry into the models was based on a probability 0.05 , and a probability of 0.10 for removal.

The project was conducted in accordance with the declaration of Helsinki and was approved by the ACT Department of Health and Community Care Human Research Ethics Committee.

\section{Results \\ Common symptoms in advanced tumours: the initial list} A list of 20 of the most common symptoms in patients with advanced tumours was generated as described earlier. The symptoms included in this list were: Lack of energy, tiredness, lack of appetite, pain, constipation, nausea, dry mouth, depression, anxiety, difficulty sleeping, tenseness, vomiting, shortness of breath, lack of sexual interest, irritability, nervousness, heartburn, dizziness, sore muscles, and numbness or tingling of the hands or feet.

\section{Prevalence rankings of symptoms by clinicians from around Australia}

Analysis of the clinicians' prevalence rankings revealed generally poor agreement among clinicians in the symptom rankings, with a maximum of only $54 \%$ of clinicians nominating the same ranking for a symptom (Table 2). Nevertheless, the clinicians tended to rank symptoms in a similar order, with the mean rankings between symptoms being moderately well separated.

Multi-dimensional scaling supported the descriptive findings, with most symptoms being ranked sequentially according to prevalence (demonstrated in Figure 1 by the spread and proximity of the symptoms to the horizontal axis). The two highest ranked (most prevalent) symptoms were lack of energy and tiredness, followed by lack of appetite and pain while the four lowest ranked (least prevalent) symptoms were numbness or tingling of the hands or feet, sore muscles, dizziness and heartburn.

A second dimension was evident in the clinicians' rankings (demonstrated in Figure 1 by the spread of symptoms along the vertical axis). Physical symptoms tended to be in the lower half of the graph while emotional symptoms tended to be in the upper half of the graph, suggesting that clinicians were not only focusing on symptom prevalence, 
Table 4: Clinicians' highest three frequencies of rankings for each symptom (percentage of clinicians giving the ranking in brackets') and mean rank (SD).

\begin{tabular}{|c|c|c|c|c|c|}
\hline Order & Symptom & | st Ranking (\%) & $2^{\text {nd }}$ Ranking (\%) & $3^{\text {rd }}$ Ranking (\%) & Mean Rank (SD) \\
\hline I & Lack of energy & I (54) & $2(25)$ & $3(9)$ & $2.16(2.37)$ \\
\hline 2 & Tired & I (39) & $2(32)$ & $3(10)$ & $2.56(2.52)$ \\
\hline 3 & Lack of appetite & $3(34)$ & $2(19)$ & $I(10)$ & $3.74(2.40)$ \\
\hline 4 & Pain & $3(17)$ & I (I7) & $4(15)$ & $4.22(3.00)$ \\
\hline 5 & Nausea & $5(17)$ & $6(14)$ & $8(11)$ & 6.77 (3.39) \\
\hline 6 & Constipation & $4(16)$ & $6(11)$ & $5(I I)$ & 7.02 (3.89) \\
\hline 7 & Anxiety & $5(13)$ & $4(13)$ & $6(10)$ & $7.34(3.60)$ \\
\hline 8 & Depressed & $5(14)$ & $7(12)$ & $4(9)$ & $7.69(3.75)$ \\
\hline 9 & Difficulty sleeping & $5(12)$ & $7(11)$ & $10(10)$ & 8.37 (3.79) \\
\hline 10 & Shortness of breath & $9(11)$ & $7(10)$ & II (9) & $9.74(4.16)$ \\
\hline II & Dry mouth & $5(8)$ & $12(8)$ & $6(7)$ & $10.17(5.04)$ \\
\hline 12 & Vomiting & $8(10)$ & $10(10)$ & $13(9)$ & 10.20 \\
\hline 13 & Tense & $6(9)$ & $13(9)$ & $9(8)$ & $10.34(4.48)$ \\
\hline 14 & Nervous & $10(9)$ & $16(9)$ & $15(8)$ & $11.27(4.69)$ \\
\hline 15 & Lack of sexual interest & $13(10)$ & $8(8)$ & $10(8)$ & $11.68(5.02)$ \\
\hline 16 & Irritability & $14(10)$ & $10(9)$ & II (9) & $11.76(4.33)$ \\
\hline 17 & Heartburn & $17(11)$ & $12(9)$ & $15(8)$ & $13.12(4.52)$ \\
\hline 18 & Dizziness & $18(13)$ & $17(12)$ & $15(12)$ & $13.96(4.10)$ \\
\hline 19 & Sore muscles & $20(21)$ & $19(12)$ & $18(11)$ & $15.14(4.65)$ \\
\hline 20 & Numbness or tingling of the hands or feet & $20(28)$ & $19(18)$ & $18(14)$ & $16.67(4.01)$ \\
\hline
\end{tabular}

I. For example, looking at the distribution of rankings for lack of energy $54 \%$ of physicians ranked it as I, $25 \%$ as 2 and $9 \%$ as 3 .

but also the underlying cause (i.e. physical versus emotional).

Based on the analysis of prevalence rankings and comments received from clinicians, modifications were made to the initial 20-item symptom list. First, coughing and problems remembering things were added to the list. Second, we agreed with comments made by clinicians that the symptoms contained in the list should be predominantly tumour-related. Thus, we excluded anxiety, lack of sexual interest, nervousness and irritability, leaving a total of 18 symptoms. The exclusion of these four symptoms was not intended to indicate they were less important, but rather in acknowledgement that other assessment tools are available for these symptoms. Nevertheless, depression and tenseness were retained in the list to provide a guide to the prevalence of emotional symptoms.

\section{Prevalence rankings of symptoms by patients at the canberra hospital}

The 44 outpatients at the Canberra Hospital were a diverse group of patients with differing diagnoses. This patient diversity was useful in examining how individual symptoms added to the explanation of variance within symptom clusters and whether the symptom list should be adapted further. The prevalence of symptoms reported by the patients ranged from $64 \%$ (lack of energy) to $16 \%$ (vomiting) (Table 3). Examining the prevalence of symptoms that appeared in both the initial 20-item list and the revised 18-item list, there was a moderately strong correlation between clinicians' prevalence rankings and the prevalence of symptoms reported by the patients (Spearman's rho $=0.67, \mathrm{p}=0.005)$. Both clinicians and patients agreed that lack of energy was the most prevalent, and that numbness or tingling of the hands or feet was relatively low in prevalence. However, examples of large disagreement occurred for lack of appetite, constipation, difficulty sleeping, dry mouth, tenseness and vomiting.

\section{Severity assessment scales}

In general, the results from the two severity assessment scales were highly correlated. However, problems remembering things, shortness of breath, and numbness or tingling of the hands or feet had relatively smaller correlations between the two scales $(0.58,0.55$, and 0.57 , respectively). The distress severity scale explained most of the variance for 14 of the 18 symptoms (Table 3 ), although the impairment of functioning scale was more important for heartburn, shortness of breath, weak or sore muscles and dry mouth. As the distress severity scale explained over $90 \%$ of the variance for the majority of symptoms, the impairment of functioning severity scale was discarded in favour of the distress severity scale.

\section{Symptom clusters}

Using the distress severity scale, four significant symptom clusters (components from the principal components analysis) were found. The four symptom clusters were 


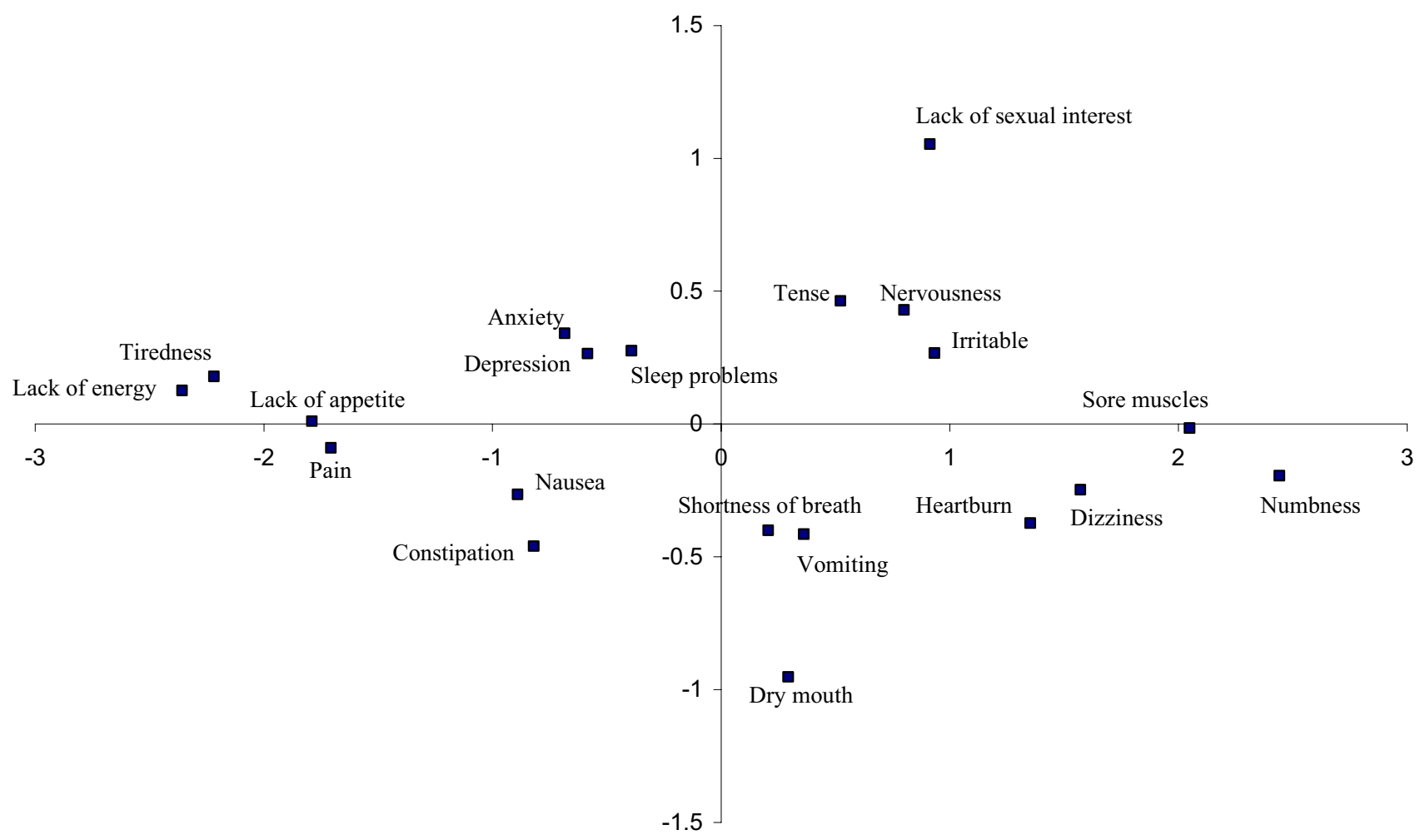

\section{Figure I}

Euclidean distance model showing the relative distances between symptoms in two dimensions, based on clinicians' prevalence rankings $(\mathrm{N}=184)$.

described as predominantly gastrointestinal (GI) symptoms (but also including pain and cognitive function), neurological/emotional symptoms, tension, and respiratory symptoms. These four clusters accounted for $69 \%$ of the variance of the varimax rotated sums of squares loadings.

Considering these four clusters as composite measures of the 18 symptoms, the variance explained by individual symptoms within a cluster was reassessed. We found that the majority of the variance in these clusters was explained by several symptoms. For example, nausea, constipation and vomiting accounted for $90 \%$ of the variance in the GI/ pain/cognitive function symptom cluster, while lack of appetite explained only $0.8 \%$ of the variance (Table 4 ). Similarly, dry mouth, heartburn, numbness or tingling of the hands or feet, depression and tiredness explained $1 \%$ or less of the variance in their respective clusters. Hence, we removed the six symptoms that explained little of the variance within their respective clusters, reducing the 18 -item symptom list to just 12 symptoms.
We decided to exclude the tension cluster because tension can be measured better using a psychological instrument, leaving 11 symptoms covering the three remaining symptom clusters. However, we decided to reinstate lack of appetite because of its strong predictive value with respect to survival [30]. Thus, we have recommended 12 symptoms that explain $97.8 \%$ of the variance in the $\mathrm{GI} /$ pain/cognitive cluster, $96.8 \%$ of the variance in the neurological/ emotional cluster, and $100 \%$ of the variance in the respiratory cluster. The final 12 symptoms, contained in these three symptom clusters (Table 5) are: Lack of energy, lack of appetite, pain, constipation, nausea, difficulty sleeping, vomiting, shortness of breath, dizziness, sore muscles, problems remembering things and coughing.

Using these 12 symptoms, we have devised a new 12-item symptom instrument ('The Canberra Symptom Scorecard') that incorporates a single distress severity scale. Included is space for patients to insert up to three additional symptoms not included in the instrument. The Canberra Symptom Scorecard is about to be assessed in centres in Australia and overseas in patients with 
Table 5: Percentage of patients that experienced each symptom and the favoured severity scale for each symptom, based on variance explained' $(\mathbf{N}=\mathbf{4 4})$.

\begin{tabular}{lllll}
\hline Ranking & Symptoms & \% With symptom & Favoured severity scale & \% Variance explained \\
\hline 1 & Lack of energy & 64 & Distress & 92 \\
1 & Pain & 64 & Distress & 95 \\
3 & Tiredness & 59 & Distress & 91 \\
4 & Difficulty sleeping & 46 & Distress & 93 \\
5 & Dry mouth & 45 & Functioning & 95 \\
6 & Tense & 43 & Distress & 94 \\
7 & Depression & 39 & Distress & 98 \\
7 & Nausea & 39 & Distress & 94 \\
7 & Problems remembering & 39 & Distress & 87 \\
10 & things & & & 76 \\
10 & Shortness of breath & 36 & Functioning & 95 \\
12 & Heartburn/belching & 36 & Functioning & 97 \\
12 & Lack of appetite & 32 & Distress & 94 \\
14 & Constipation & 32 & Distress & 99 \\
15 & Dizziness & Distress & 99 \\
16 & Weak or sore muscles & 27 & Functioning & 81 \\
17 & Numbness or tingling of & 25 & Distress & 95 \\
18 & the hands or feet & 23 & Distress & 96
\end{tabular}

I. Variance explained refers to the adjusted $\mathrm{R}^{2}$ obtained from OLS regressions where the dependent variable was the sum of scores from the two severity scales (distress \& function) for a symptom, and the explanatory variables were the severity scales.

Table 6: Selected results from stepwise ordinary least squares regressions showing the percentage of variance explained for each symptom associated with the cluster symptom measure'.

\begin{tabular}{|c|c|c|c|c|c|c|c|}
\hline Cluster I GI/pain/cogn & $\%$ Var & Cluster 2 Neural/emot & $\%$ Var & $\begin{array}{l}\text { Cluster } 3 \\
\text { Tension }\end{array}$ & $\%$ Var & $\begin{array}{l}\text { Cluster } 4 \\
\text { Respiratory }\end{array}$ & $\%$ Var \\
\hline Vomiting & 5.5 & Difficulty sleeping & 19.7 & Tense & 70.3 & Coughing & 18.9 \\
\hline Lack of appetite & 0.8 & Dizziness & 66.8 & $\begin{array}{l}\text { Shortness of } \\
\text { breath }\end{array}$ & 29.7 & $\begin{array}{l}\text { Shortness of } \\
\text { breath }\end{array}$ & 81.1 \\
\hline Nausea & 69.9 & Sore/weak muscles & 5.4 & & & breath & \\
\hline Dry mouth & 1.0 & Numbness & 1.0 & & & & \\
\hline Constipation & 14.6 & Depression & 1.4 & & & & \\
\hline Heartburn & 1.3 & Tiredness & 0.9 & & & & \\
\hline Pain & 3.3 & Lack of energy & 4.9 & & & & \\
\hline Problems remembering things & 3.7 & & & & & & \\
\hline
\end{tabular}

I. A composite symptom measure was created for each of the four clusters (Gl/pain/cognitive, Neurological/emotional, Tension, and Respiratory). The symptoms associated with each composite measure were then examined in a stepwise method for their contribution to explaining the variation in each cluster. The order symptoms were entered into the regression model follows the amount of variance explained, with the largest being entered first.

advanced (incurable) tumours receiving palliative treatments. As part of this next step in the development of The Canberra Symptom Scorecard, it will be validated against selected existing instruments.

\section{Discussion}

The findings of this study suggest that the initial 20-item symptom list provided a reasonable representation of the symptoms experienced by patients with advanced tumours (Table 1). However, the comments received from the clinicians yielded two additional symptoms (problems remembering things and coughing) that proved to be highly prevalent in the patients with incurable tumours surveyed (39\% and 23\% respectively). The other important suggestion provided by the clinicians was to focus on physical symptoms due to the tumour rather than emotional 
Table 7: Symptom clusters and symptoms in the canberra symptom scorecard

\begin{tabular}{ll}
\hline Symptom Cluster & Symptoms \\
\hline GI/pain/cognitive & I Nausea \\
& 2 Constipation \\
& 3 Vomiting \\
& 4 Lack of appetite \\
& 5 Pain \\
& 6 Problems remembering things \\
& 7 Dizziness \\
& 8 Problems sleeping \\
& 9 Weak or sore muscles \\
& I0 Lack of energy \\
& II Shortness of breath \\
& 12 Coughing \\
\end{tabular}

symptoms, since other instruments assess these better. Consequently, we reduced the list to 18 symptoms that focused predominantly on physical problems.

The high prevalence rates for all 18 symptoms in the 44 outpatients with incurable tumours surveyed further supported the coverage (Table 2). However, these high rates made it difficult to identify individual symptoms that could be eliminated from the list, as we had aimed to identify only those symptoms essential for routine measurement.

We were able to reduce the number of symptoms further, while maintaining good coverage, by exploring symptom clusters. Eleven symptoms were found to explain adequately the variation within the three clusters that covered the physical symptoms (Table 4). However, we added lack of appetite to the symptom list, making a final list of 12 symptoms. Our findings are consistent with those of Watson et al [29] who also identified similar broad clusters of physical symptoms in cancer patients, using a 30item version of the RSCL. We believe that these three clusters (Table 5) encompass the most important symptoms in patients with advanced tumours.

Using this final list of 12 symptoms as a guide, we reassessed existing symptom assessment instruments and found that some did not address all the symptoms we recommend (e.g. ESAS, MDASI), while others covered too many symptoms, making them too lengthy for regular administration (e.g. ASDS, MQOL, MSAS, RSCL). For example, the core list of 13 symptoms of the MDASI [13] is limited in the assessment of neurological symptoms but contains a considerable number of symptoms related to emotional problems. In our opinion, the usefulness of assessing emotional (or psychological) symptoms this way is questionable. The CSS does not include assessment of emotional or psychological symptoms, as it concentrates on physical symptoms related to the tumour. If assessment of emotional symptoms is also required, we recommend that another suitable instrument be used.

In summary, despite a relatively large number of available symptom assessment instruments, little research has been undertaken to determine the adequate coverage of symptom clusters appropriate for patients with advanced tumours, and consequently the minimum list of symptoms needed to measure these clusters. In the present study, we undertook such an investigation that yielded three important symptom clusters and their associated symptoms. Comparing our final list and existing symptom lists, we found major symptoms were missing from shorter lists most likely to be considered for routine measurement in clinical practice. As a result, we recommend that modifications need to be made to existing instruments to address these limitations, and that further consideration be given to issues around the measurement of physical symptoms and psychological symptoms.

Based on our findings, we have identified a brief set of symptoms covering the common physical symptoms experienced by outpatients with advanced tumours. From this we have developed a symptom assessment instrument called 'The Canberra Symptom Scorecard' (CSS) that comprehensively covers the important and common symptoms associated with advanced (incurable) tumours. We believe that this instrument should help clinicians to monitor these symptoms in outpatients undergoing palliative treatment(s), such as palliative chemotherapy, palliative radiotherapy or symptomatic treatments. It is about to be assessed in patients with advanced tumours receiving palliative treatments in centres in Australia and overseas, and, as part of this assessment, it will be validated against existing instruments. We believe that this investigation should establish the validity and usefulness of the CSS in patients with advanced tumours receiving palliative treatments aimed at improving patients' symptoms.

\section{Conclusions}

We reviewed existing symptom assessment instruments and found a number of deficiencies such as existing instruments being too long or burdensome, too short, or measuring quality of life, rather than tumour-related physical symptoms. Others sacrificed important physical symptoms for emotional symptoms that may be assessed better using psychological instruments. Therefore, we devised a new symptom instrument ("The Canberra Symptom Scorecard", (CSS)). This is a short 12-item symptom questionnaire that focuses on the physical symptoms important in patients with advanced tumours. It appears suitable for outpatients with advanced (incura- 
ble) tumours receiving palliative treatments and is about to be assessed in such patients, both in Australia and overseas when it will also be validated against existing instruments.

\section{Competing interests}

None declared.

\section{Authors' contributions}

All authors participated equally in this research project. MJB conducted the interviews with patients and clinicians and drafted the manuscript. BS was responsible for the analysis of the results. RSH, DB and BS conceived the study and participated in its design and coordination. All authors read and approved the final manuscript.

\section{Additional material}

\section{Additional File 1}

Table 1 - Self-Report Quality of Life and Functional Instruments to Measure Symptom Occurrence and/or Distress in Adult Patients with Advanced Tumours.

Click here for file

[http://www.biomedcentral.com/content/supplementary/14712407-3-32-S1.doc]

\section{Additional File 2}

Table 3-Q1 During the past 7 days including today, have you suffered from a lack of energy?

Click here for file

[http://www.biomedcentral.com/content/supplementary/1471-

2407-3-32-S2.doc]

\section{Acknowledgements}

We would like to thank The Canberra Hospital Private Practice Fund for providing a research grant that supported this investigation. We would also like to thank all the clinicians and patients who participated in the study and those clinicians who referred patients into the study.

\section{References}

I. Bruera E, Kuehn N, Miller MJ, Selmser P, Macmillan K: The Edmonton symptom assessment system (ESAS): a simple method for the assessment of palliative care patients. J Palliat Care I99I, 7:6-9.

2. Hollen PJ, Gralla RJ, Kris MG, Potanovich LM: Quality of life assessment in individuals with lung cancer: testing the lung cancer symptom scale (LCSS). Eur J Cancer 1993, 29A(Suppl I):S5I-S58.

3. Holmes $S$ : Use of a modified symptom distress scale in assessment of the cancer patient. Int J Nurs Stud 1989, 26:69-79.

4. McCorkle R, Young K: Development of a symptom distress scale. Cancer Nurs 1978, I:373-378.

5. McCorkle R, Quint-Benoliel J: Symptom distress, current concerns and mood disturbance after diagnosis of life-threatening disease. Soc Sci Med 1983, 17:431-438.

6. Rhodes VA, Watson PM, Johnson MH: Development of reliable and valid measures of nausea and vomiting. Cancer Nurs 1984, 7:33-4I.

7. Cella DF, Tulsky DS, Gray G, Sarafian B, Linn E, Bonomi A, Silberman $M$, Yellen SB, Winicour P, Brannon J: The functional assessment of cancer therapy scale: development and validation of the general measure. J Clin Oncol 1993, I I:570-579.

8. Higginson IJ, McCarthy M: Validity of the support team assessment schedule: do staff' ratings reflect those made by patients or their families? Palliat Med 1993, 7:219-228.

9. Higginson IJ, McCarthy M: A comparison of two measures of quality of life: their sensitivity and validity for patients with advanced cancer. Palliat Med 1994, 8:282-290.

10. Lo RS, Ding A, Chung TK, Woo J: Prospective study of symptom control in 133 cases of palliative care inpatients in Shatin hospital. Palliat Med 1999, I 3:335-340.

II. Cohen SR, Mount BM, Strobel MG, Bui F: The McGill quality of life questionnaire: a measure of quality of life appropriate for people with advanced disease. A preliminary study of validity and acceptability. Palliat Med I995, 9:207-2I9.

12. Portenoy RK, Thaler HT, Kornblith AB, Lepore JM, Friedlander-Klar H, Kiyasu E, Sobel K, Coyle N, Kemeny N, Norton L et al.: The Memorial symptom assessment scale: an instrument for the evaluation of symptom prevalence, characteristics and distress. Eur J Cancer I994, 30A: I326-1336.

13. Cleeland CS, Mendoza TR, Wang XS, Chou C, Harle MT, Morrissey $M$, Engstrom MC: Assessing symptom distress in cancer patients: the M.D. Anderson symptom inventory. Cancer 2000, 89: 1634-1646.

14. De Haes JC, van Knippenberg FC, Neijt JP: Measuring psychological and physical distress in cancer patients: structure and application of the Rotterdam Symptom Checklist. $\mathrm{Br}$ J Cancer 1990, 62:1034-1038.

15. Richards MA, Ramirez AJ: Quality of life: the main outcome measure of palliative care. Palliat Med I 997, I I:89-92.

16. Kramer JA, Curran D, Piccart M, de Haes JC, Bruning P, Klijn J, Van Hoorebeeck I, Paridaens R: Identification and interpretation of clinical and quality of life prognostic factors for survival and response to treatment in first-line chemotherapy in advanced breast cancer. Eur J Cancer 2000, 36: | 498 - I506.

17. Kramer JA, Curran D, Piccart M, de Haes JC, Bruning PF, Klijn JG, Bontenbal M, van Pottelsberghe C, Groenvold M, Paridaens R: Randomised trial of paclitaxel versus doxorubicin as first-line chemotherapy for advanced breast cancer: quality of life evaluation using the EORTC QLQ-C30 and the Rotterdam symptom checklist. Eur J Cancer 2000, 36: I488- I 497.

18. Hardy JR, Edmonds P, Turner R, Rees E, A'Hern R: The use of the Rotterdam symptom checklist in palliative care. J Pain Symptom Manage 1999, I 8:79-84.

19. Lee WR, McQuellon RP, McCullough DL: A prospective analysis of patient-reported quality of life after prostate brachytherapy. Semin Urol Oncol 2000, I8(2): I45-I5I.

20. Kearsley JH, Schonfeld C, Sheehan M: Quality-of-life assessment during palliative radiotherapy. Australas Radiol I998, 42:354-359.

21. Rees E, Hardy J, Ling J, Broadley K, A'Hern R: The use of the Edmonton symptom assessment scale (ESAS) within a palliative care unit in the U.K. Palliat Med 1998, I 2:75-82.

22. Pratheepawanit N, Salek MS, Finlay IG: The applicability of quality-of-life assessment in palliative care: comparing two quality-of-life measures. Palliat Med I999, I 3:325-334.

23. Bruera E, Neumann C, Brenneis C, Quan H: Frequency of symptom distress and poor prognostic indicators in palliative cancer patients admitted to a tertiary palliative care unit, hospices, and acute care hospitals. J Palliat Care 2000, I6:16-2I.

24. Rathbone GV, Horsley S, Goacher J: A self-evaluated assessment suitable for seriously ill hospice patients. Palliat Med 1994, 8:29-34.

25. Cohen SR, Mount BM, Bruera E, Provost M, Rowe J, Tong K: Validity of the McGill quality of life questionnaire in the palliative care setting: a multi-centre Canadian study demonstrating the importance of the existential domain. Palliat Med 1997, I I:3-20.

26. Donnelly S, Walsh D: The symptoms of advanced cancer. Semin Oncol 1995, 22(2 Suppl 3):67-72.

27. Walsh D, Donnelly S, Rybicki L: The symptoms of advanced cancer: relationship to age, gender, and performance status in I,000 patients. Support Care Cancer 2000, 8: I75-I79.

28. Carroll BT, Kathol RG, Noyes R Jr, Wald TG, Clamon GH: Screening for depression and anxiety in cancer patients using the hospital anxiety and depression scale. Gen Hosp Psychiatry 1993, 15:69-74. 
29. Watson M, Law GP, Maguire B, Robertson B, Greer S, Bliss M, Ibbotson $\mathrm{T}$ : Further development of a quality of life measure for cancer patients: the Rotterdam symptom checklist (revised). Psychooncology 1992, 1:35-44.

30. Morita T, Tsunoda J, Inoue S, Chihara S, Ishimoto O, Hisaoka N, Itoh M: Prediction of survival of terminally ill cancer patients - a prospective study. Gan to Kagaku Ryoho 1998, 25: I 203-I2I I.

\section{Pre-publication history}

The pre-publication history for this paper can be accessed here:

http://www.biomedcentral.com/1471-2407/3/32/prepub

Publish with Bio Med Central and every scientist can read your work free of charge

"BioMed Central will be the most significant development for disseminating the results of biomedical research in our lifetime. "

Sir Paul Nurse, Cancer Research UK

Your research papers will be:

- available free of charge to the entire biomedical community

- peer reviewed and published immediately upon acceptance

- cited in PubMed and archived on PubMed Central

- yours - you keep the copyright

Submit your manuscript here:

http://www.biomedcentral.com/info/publishing_adv.asp 